\title{
Distale Femurfrakturen
}

\author{
Uwe Frerichmann, Alex Rübberdt, Michael J. Raschke
}

\section{Zusammenfassung}

Durch die modernen minimalinvasiven Operationsverfahren mittels LISS und retrogradem Femurnagel konnten einerseits Komplikationsraten in der Behandlung distaler Femurfrakturen gesenkt werden, andererseits werfen die neuen OP-Verfahren auch neue, bisher nicht gekannte, Probleme auf, die die operative Vorsorgung distaler Femurfrakturen zu einem anspruchsvollen Eingriff machen.

Das Spektrum der distalen Femurfrakturen erstreckt sich von (osteoporosebedingten) per-/suprakondylären Frakturen über periprothetische Frakturen bis hin zu schweren hoch enenergetischen Verletzungsbildern des distalen Femurs häufig bei jüngeren, meist polytraumatisierten Patienten. Diese können prinzipiell sowohl mit winkelstabilen internen Fixationssystemen (z. B. LISS) als auch mit retrograden Femurnägeln stabilisiert werden. Das LISS zeichnet sich durch einen breiteren Einsatzbereich sowie einer stabilen Verankerung mit höherer Rotationsstabilität, insbesondere im osteoporotischen Knochen aus. Zusätzlich wird die Gefahr einer intraoperativen Sprengung des Kondylenmassivs vermieden. Typische Komplikationen der LISS sind proximale Schraubenausrisse durch intraoperati-

\section{Einleitung}

Die Versorgung distaler Femurfrakturen ist trotz zahlreicher innovativer Implantateentwicklungen technisch sehr an-

OP-JOURNAL 2005; 21: 160-170

(C) Georg Thieme Verlag KG Stuttgart · New York am Femurschaft. Lösungsoptionen bieten die Verwendung langer Plattensysteme und die bikortikale proximale Schaftverankerung. Weitere Probleme sind Varus- und Valgus- sowie Rotationsfehler, Traktusirritationen, sowie das Auftragen des Implantates, besonders bei schlanken Patienten.

Der retrograde Femurnagel kommt häufig bei jungen und adipösen $\mathrm{Pa}$ tienten mit extraartikulärer oder gering dislozierter intraartikulärer Fraktur des distalen Femurs zum Einsatz.

Implantatbedingte Kompliationen sind u. a. bedingt durch einen falschen Nageleintrittspunkt, die Verwendung eines zu kurzen Nagels oder das Auftreten eines Notchimpingements bei einem in das Kniegelenk hineinreichenden Nagel. Varus-/Valgusfehlstellungen im metaphysären Bereich sind nicht selten zu beobachtende Komplikationen. In Einzelfällen wurde die Sprengung des unverletzten Kondylenmassivs beim Einschlagen eine retrograden Nagels beschrieben.

Bei gleichzeitig vorliegender Kreuzbandverletzung ist die Verwendung eines retrograden Nagels kritisch zu prüfen, da hierdurch unter Umständen eine (zeitnahe) operative Vorsorgung deutlich erschwert ist. ve Fehlplatzierung der proximalen LISS

$[19,23]$ ist mit einer hohen Zahl an Revisionsoperationen aufgrund Repositionsverlust, Implantatversagen und ausbleibender Frakturheilung verbunden [17] Durch die Entwicklung indirekter Repositionsmethoden sowie minimalinvasiver Operationstechniken konnte die Häufigkeit dieser Komplikationen reduziert werden [10], andererseits werfen die neuen OP-Verfahren auch neue, bisher nicht gekannte Probleme auf, die trotz korrekter Einstellung der Achsverhältnisse, die Therapie distaler Femurfrakturen zu einer anspruchsvollen Operation machen.

\section{Klassifikation}

Die AO-Klassifikation wird einer Unterscheidung in intra- und extraartikuläre Verletzung, welche für die Therapie und Prognose der distalen Femurfraktur von entscheidender Bedeutung ist, am besten gerecht (Abb.1).

A-Frakturen sind suprakondyläre, extraartikuläre Frakturen, B-Frakturen verlaufen partiell intraartikulär und C-Frakturen sind vollständig intraartikuläre Frakturen.

Die Hoffa-Fraktur (AO 33-B3-Fraktur) ist eine isolierte tangentiale und in der Frontalebene verlaufende Fraktur der dorsalen Femurkondyle, häufig im Rahmen eines Polytraumas und kann schnell übersehen werden. Bei fehlender adäquater Therapie resultiert eine Pseudarthrose oder aufgrund der Valgisierung bzw. Varisierung des Kniegelenkes eine posttraumatische Arthrose [16]. me bezüglich Achsfehlstellung, Pseudarthrosenrate, sekundärer Dislokation und Infektrate auf [2].

Oberstes Behandlungsziel ist die Wiederherstellung der Achsverhältnisse und die Rekonstruktion des Kondylenmassivs.

Die offene Reposition und konventionelle Plattenosteosynthese dieser Frakturen

\section{Verletzungsmechanismus}

Bei den distalen Femurfrakturen werden zwei Verletzungsmechanismen unterschieden:

Die Niedrigernergietraumen treten vorwiegend bei älteren Patienten mit Osteoporose auf oder sind mit periprotheti- 


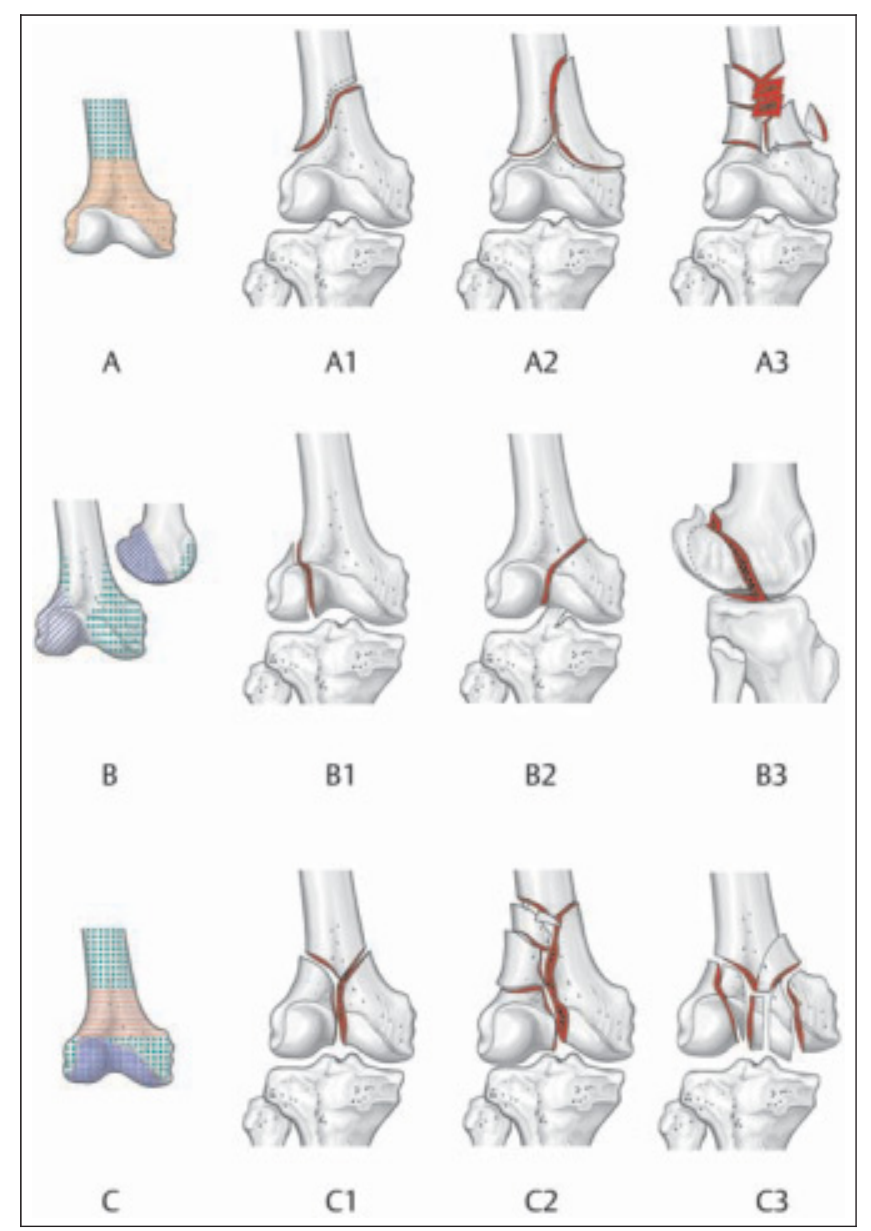

schen Frakturen verbunden. Handelt es sich um eine periprothetische Fraktur muss geklärt werden, ob radiomorphologische Zeichen einer Prothesenlockerung vorliegen, um einer einzeitigen Frakturversorgung und Prothesenwechsel durchführen zu können.

Das Kernproblem bei der osteosynthetischern Versorgung ist die sichere Verankerung des Implantates im osteoporotischen Knochen.

In Abhängigkeit vom verwendeten Prothesentyp ist der Implantatwahl Grenzen gesetzt.

Die Hochrasanztraumen treten vorwiegend bei jungen Patienten auf und sind häufig mit Trümmerfrakturen der Kondylen- und Metaphysenregion sowie mit schweren Weichteilschäden verbunden. Neben Knorpelverletzungen kommt es oft auch zu komplexen Kniebandverletzungen, so dass ein primäres Behandlungsziel die Wiederherstellung der Funktion eines zerstörten Kniegelenks darstellt [21].
Abb.1 AO-Klassifikation distaler Femurfrakturen.
Hinweisen für eine Kniegelenksluxation oder floating knee-Verletzung ist eine zeitnahe Doppler-/Duplex-Sonographie durchzuführen.

Die radiologische Basisdiagnostik beinhaltet eine Zielaufnahme des Kniegelenks mit angrenzendem Femur in 2 Ebenen.

Eine primäre CT-Untersuchung liefert wertvolle Zusatzinformationen bei komplexen Frakturmustern und bietet die Option, durch Verwendung von Kontrastmittel Gefäßverletzungen im POP-I bis III-Segment sicher abzubilden. Das MRT spielt in der Primärdiagnostik eine untergeordnete Rolle.

\section{Therapie}

Die konservative Therapie mit langfristiger Immobilisation im Oberschenkelliegegips bzw. Tutor [19] sollte vermieden werden. Die operative Versorgung distaler Femurfrakturen stellt heutzutage den Goldstandard dar. Die Art der Frakturversorgung orientiert sich am Frakturtyp (AO-Klassifikation), an der Schwere des Weichteilschadens, lebensbedrohlicher Begleitverletzungen oder Faktoren wie osteopene Knochenstruktur oder liegende Implantate $[18,21]$.

Die offene Reposition und Plattenosteosynthese mittels $95^{\circ}$ Kondylenplatte oder dynamischer Kondylenschraube (DCS) waren lange Zeit die Standardimplantate. Beide Systeme bieten neben einer guten Rotationsstabilität die Vorteile einer winkelstabilen Verankerung im Kondylenmassiv [8]. Beide Verfahren sind technisch sehr anspruchsvoll, bieten wenig Korrekturoptionen und verzeihen keine operativer Fehler. Sowohl die $95^{\circ}$ Kondylenplatte als auch die DCS sind gegenwärtig Implantate der 2 . Wahl bei der operativen Versorgung von distalen Femurfrakturen. Gefäßverletzung (kalter Unterschenkel oder Fuß) bzw. klinisch/anamnestischen
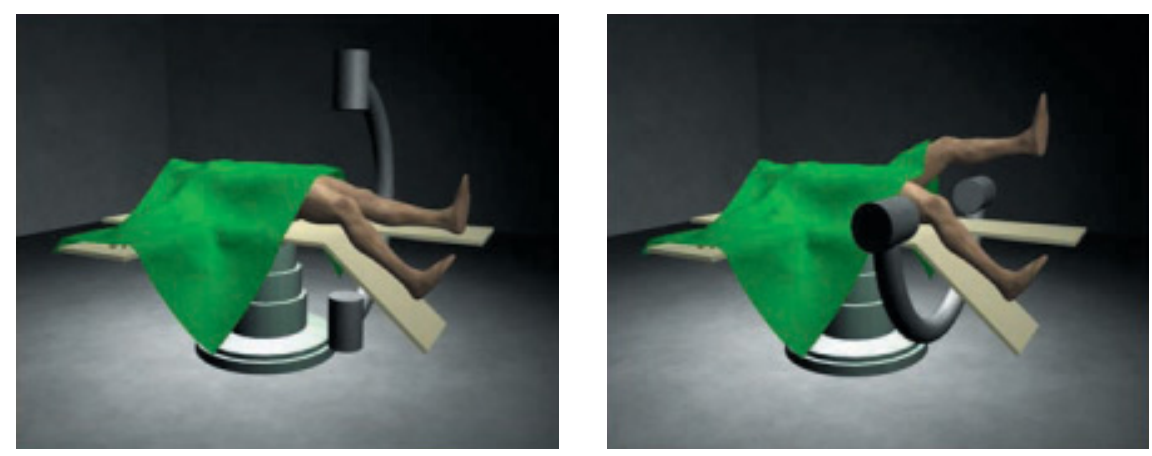

Abb. 2 Lagerung des Patienten mit Möglichkeit der intraoperativen Durchleuchtung in 2 Ebenen. 
Die retrograde Femurnagelung und LISSOsteosynthese sind heutzutage die Standardverfahren. Auf sie wird im Folgenden dezidiert eingegangen. Der Vollständigkeit halber werden die Verfahren der Schrauben-/Plattenosteosynthesen und der Fixateur-externe-Behandlung mit aufgeführt.

\section{Patientenlagerung}

Es empfiehlt sich eine Rückenlagerung des Patienten auf einem röntgendurchlässigen Operationstisch. Besondere Vorteile bieten hier Carbontische oder Operationstische mit Carbon-Ansteckplatten (CFK ${ }^{\circledR}$, Fa. Maquet). Die verletzte Extremität sollte frei gelagert sein. Bei der retrograden Femurmarknagelung bietet sich die Verwendung einer sterilen Knierolle oder steril eingepackten Kniekissen an. Alternativ kann der Unterschenkel der verletzen Extremität in $45^{\circ}$ - bis $90^{\circ}$-Position abgesenkt und das Einschlagen des Marknagels in dieser Position durchgeführt werden (Abb.2). Zur einfacheren Durchleuchtung kann die unverletzte Seite abgesenkt oder die verletzte Seite angehoben werden.

\section{A) Retrograde Marknagelung}

Die Indikation zur retrograden Marknagelung (Abb.3) besteht bei jungen Patienten mit extraartikulärer Fraktur ( $\mathrm{AO}$ $33 \mathrm{~A})$ sowie bei gering dislozierten intraartikulären Frakturen (AO $33 \mathrm{C} 1$ und $\mathrm{C} 2$ ) $[3,6,15]$.

Die Wahl des Zugangsweges ist abhängig vom Frakturtyp. In der Regel wird ein infrapatellarer transligamentärer Zugang gewählt. Bei intraartikulären Frakturen vom Typ AO 33 C1 und C2 empfiehlt sich eine primäre perkutane Schrauben-
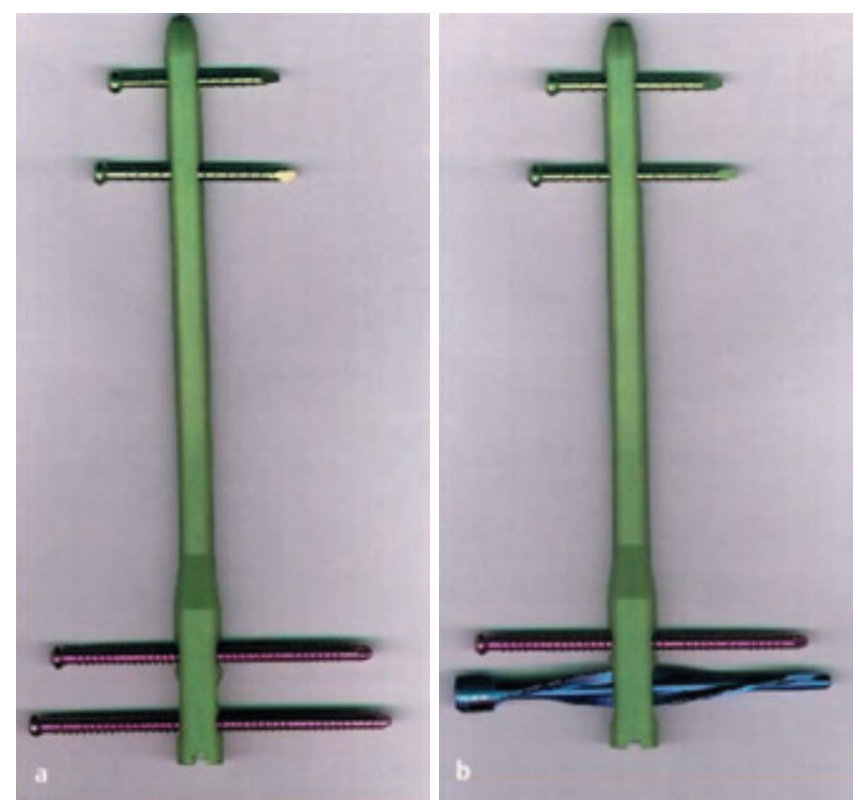

Abb. 3 (a) Distaler Femurnagel (DFN), (b) Distaler Femurnagel mit Spiralklinge. osteosynthese (3,5 oder 6,5 mm Schrauben) des mittels Repo-Zange retinierten Kondylenmassivs. Bei der Schraubenplatzierung im Kondylenmasiv ist der späteren Lage des Marknagels Respekt zu zollen. Eine aufwändige intraartikuläre Rekonstruktion verlangt in der Regel eine Arthrotomie (Abb.4).

Der knöcherne Eintrittspunkt des retrograden Marknagels liegt in Verlängerung der Femurschaftachse und $0,5-1 \mathrm{~cm}$ oberhalb der femoralen Insertion des hinteren Kreuzbands (Abb.5).

Zur genauen Festlegung des Nageleintrittspunktes empfiehlt sich die Verwendung eines Bildwandlers. Der Markraum wird mittels Pfriem oder kanüliertem Bohrer nach Plazierung eines K-Drahtes eröffnet. Bei kanülierter Technik wird ein Führungsdraht unter BV-Sicht über die Fraktur geschoben und die Nagellänge bestimmt (Abb.6). Gegebenenfalls wird der Femurschaft über den Führungsdraht aufgebohrt. Eine andere Möglichkeit ist den Nagel in unaufgebohrter Technik einzubringen. Dabei kann die Fraktur direkt über den Marknagel reponiert werden.

Das distale Nagelende darf keinesfalls in das Kniegelenk hinein ragen, um ein femorales Notch-Impingement zu vermeiden.

Bei Achsabweichungen kann zur intramedullären Schienung des Nagels eine Pollerschraube eingesetzt werden. Nach Einbringen des Nagels erfolgt zunächst die distale Verriegelung. Anschließend erfolgt die klinische und radiologische Kontrolle der Reposition, der Rotation und der Beinläge. Abschließend wird
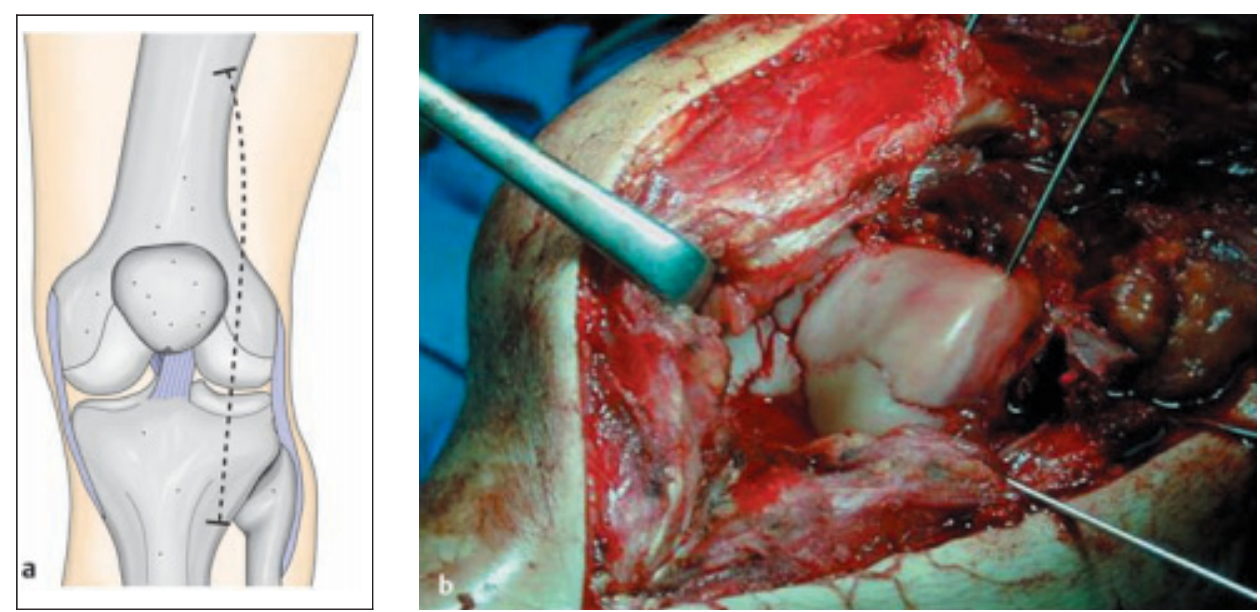

Abb. 4 (a) Parapatellare Arthrotomie bei (b) notwendiger Rekonstruktion der Gelenkfläche. 


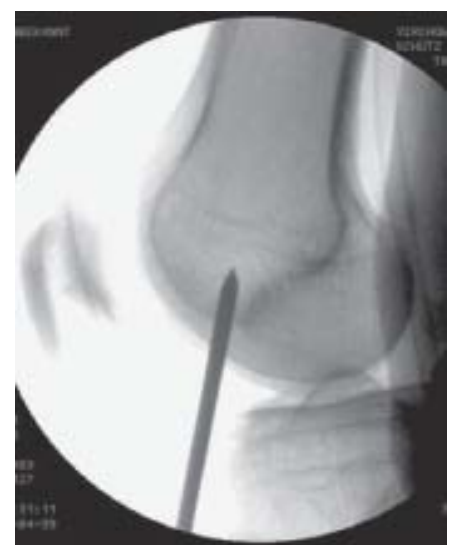

Abb. 5 Eintrittspunkt Retrograder Marknagel.
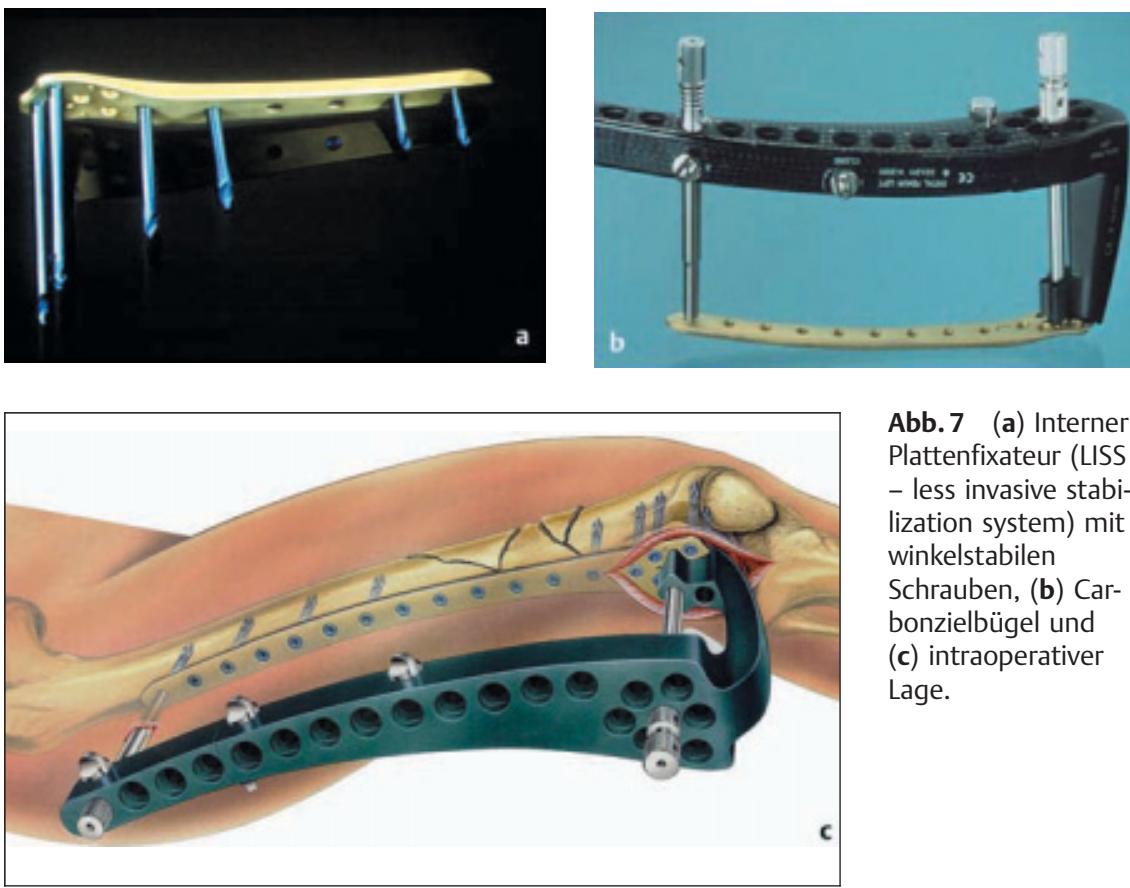

Abb. 7 (a) Interner Plattenfixateur (LISS - less invasive stabilization system) mit winkelstabilen

Schrauben, (b) Carbonzielbügel und

(c) intraoperativer Lage.

die proximale Verriegelung in Freihand(röntgendurchlässiges Winkelgetriebe) oder Powerdrivetechnik (direktes Eindrehen der selbstschneidenden Verriegelungsschrauben ohne Vorbohren) durchgeführt. Eine prinzipielle Empfehlung ist die Verwendung von möglichst langen Nägeln mit mindestens einfach proximaler statischer Verriegelung. Anzustreben ist die Verwendung möglichst langer Nägel.

Implantatspezifische Komplikationen sind die Wahl des falschen Nageleintrittspunktes, femorales Notch-Impingements, primäre und sekundäre Achsfehlstellung in metaphysärem Bereich. Die Lockerung und Dislokation der Verriegelungsbolzen werden insbesondere bei osteoporotischer Knochenstruktur beobachtet.
In tierexperimentellen Untersuchungen konnte eine Verminderung der Durchblutung der Kreuzbänder nach retrograder Marknagelung beobachtet werden [1] Die befürchtete akzidentielle Verletzung der Kreuzbänder wurde in der aktuellen Literatur bisher nicht beschrieben. Eine seltene Komplikation ist die intraoperative Sprengung des Kondylenmassivs beim Einschlagen des Marknagels [15].

\section{B. Winkelstabiles internes \\ Fixationssystem (z.B. LISS - less invasive stabilization system)}

Es handelt sich um einen anatomisch vorgeformten winkelstabilen inneren Plattenfixateur, bei dem selbstbohrende oder selbstschneidende Schrauben über einen röntgendurchlässigen Carbonbügel
Abb. 6 Einbringen des retrograden Marknagels. perkutan eingebracht werden (Abb.7) Die eingeschobene minimalinvasive OPTechnik mit winkelstabilen Bolzen hat eine Lernkurve und bedingt eine spezielle Operationstechnik [22]. Die metaphysär oder kortikal platzierten Schrauben verbinden sich über ein Gewinde am Außenrand des Schraubenkopfes stabil mit einem Gewinde im Plattenloch. Hierdurch wird die Winkelstabilität erreicht, die besonders bei osteoporotisch vorgeschädigtem Knochen erhebliche Vorteile aufweist (Abb. 8). Ist die Platte jedoch erst einmal am Schaft mit den winkelstabilen Bolzen fixiert, sind die Korrekturoptionen sehr begrenzt. Durch die Winkelstabilität zwischen Schrauben und Platte ist ein Anpressen der Platte auf den Knochen nicht mehr notwendig. Hierdurch wird die kortikale Durchblutung erhalten.

Voraussetzung einer gelungenen Operationstechnik ist jedoch die korrekte Lage dieser anatomisch vorgeformten Implantate.

Zusätzlich legt die Richtung des Gewindes in der Platte die spätere Richtung

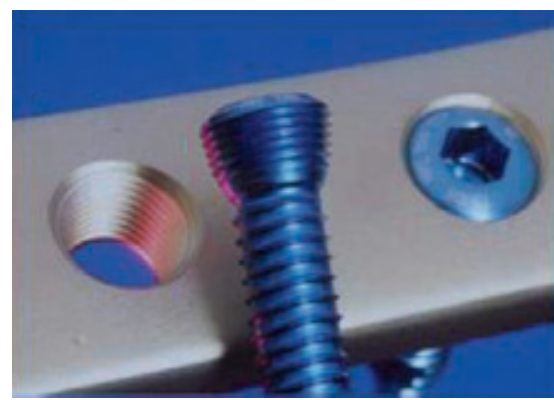

Abb. 8 Kopfverriegelnde Schraube: Die Schrauben verbinden sich über ein Gewinde am Außenrand des Schraubenkopfes winkelstabil mit einem Gewinde im Plattenloch. 


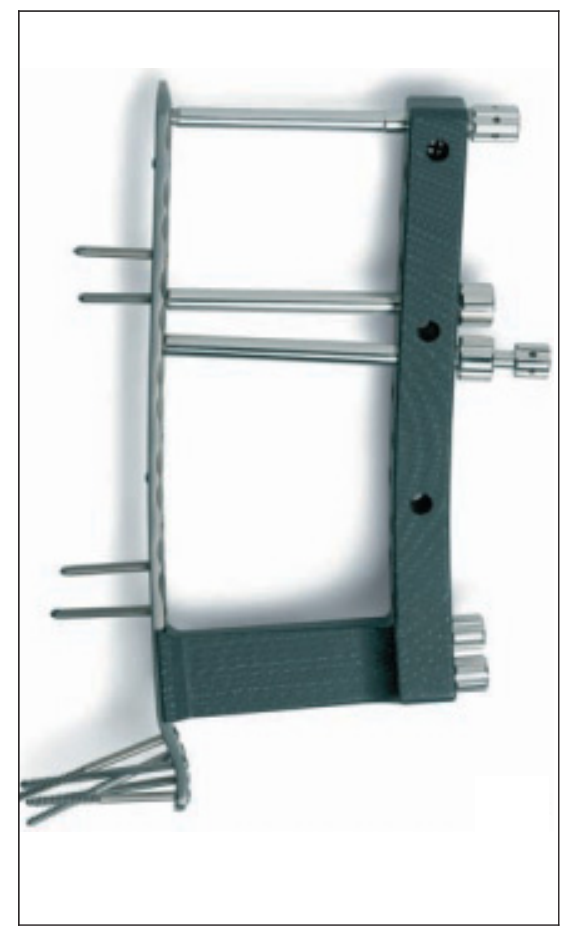

Abb. 9 NCB System (ZIMMER) mit polyaxialer Schraubenausrichtung, mit Zielbügel.

der Schraube fest. Wünschenswert sind hier neuere Systeme, bei denen die Schraubenrichtung in einem gewissen Rahmen $\left(20-30^{\circ}\right)$ polyaxial einsetzbar ist und Winkelstabilität erhalten bleibt. Dieses wird in anderen Systemen, die gerade in der ersten klinischen Erprobung sind, verwirklicht. (NCBF-System - Fa. ZIMMER) (Abb.9).

Die LISS bietet Vorteile beim osteoporotischen Patienten, da durch die Besetzung aller distalen 7 Schrauben im Bereich des Kondylenmassivs durch die größere Kontaktfläche eine bessere Verankerung ermöglicht wird, die die Gefahr der Auslockerung insbesondere im osteoporotischen Knochen vermindert.

Die Zugangswahl wird auch hier durch den Frakturtyp bestimmt.

Bei extraartikulären (AO 33 A) oder gering dislozierten intraartikulären Frakturen (AO 33 C1 und C2) erfolgt die Implantation über einen minimalinvasiven lateralen Zugang (Abb.10) [11]. Die Schnittführung erfolgt von distal nach proximal, in Gelenkhöhe beginnend, im Verlauf des Femurschaftes. Anschließend wird der M. vastus lateralis untertunnelt und das Plattensystem mit dem Zielbügel eingeschoben. Bei dislozierten intraartikulären Frakturen (AO 33 C3) erfolgt eine parapa-

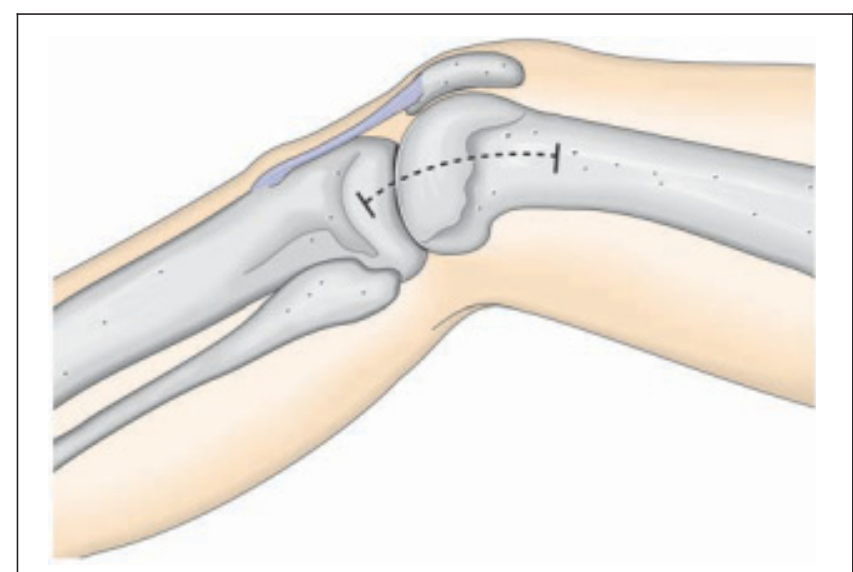

Abb.10 Minimalinvasiver lateraler Subvastuszugang.
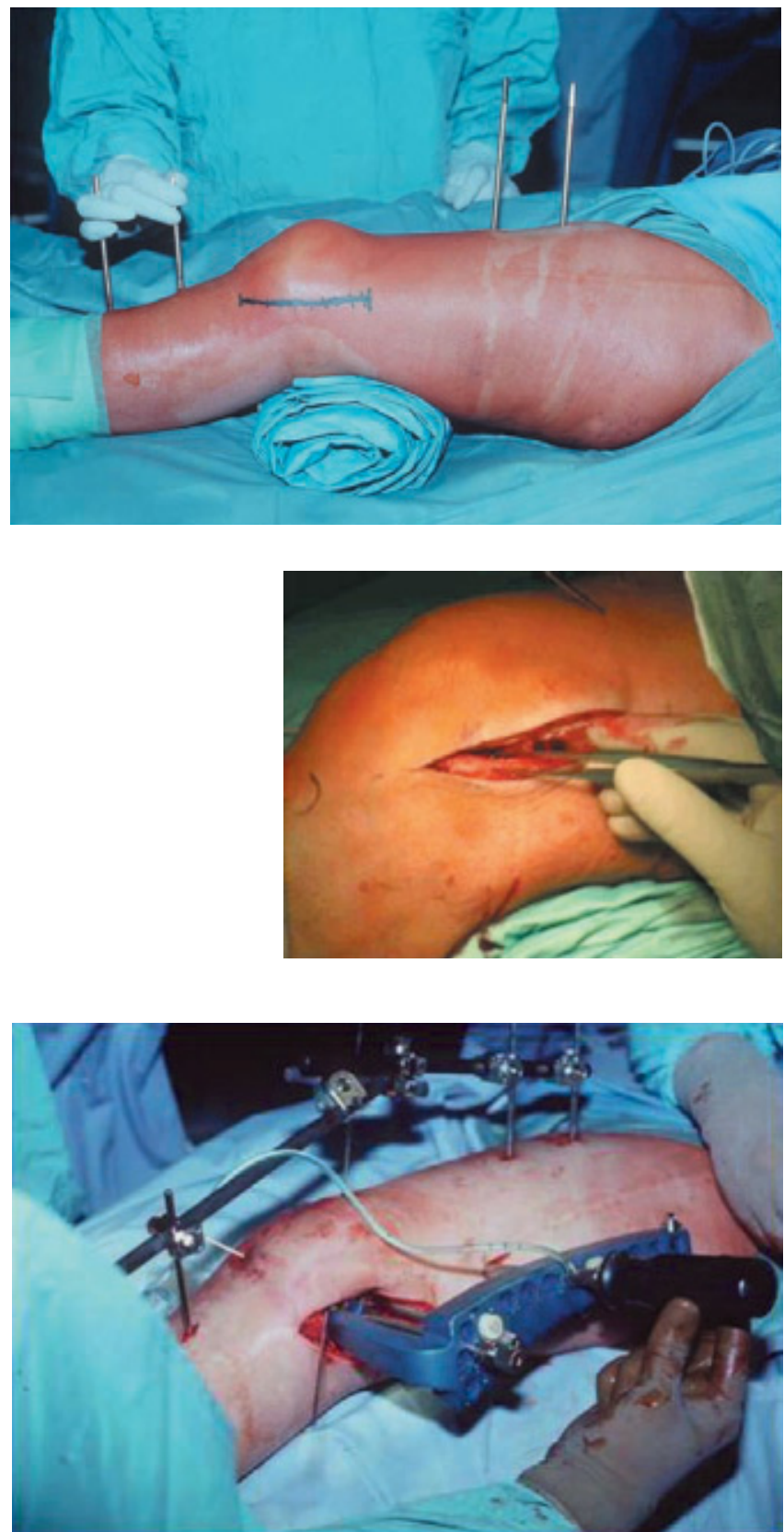

Abb.11 Reposition des Gelenkblocks mit dem Femurschaft mit temporärer externer Stabilisierung. 

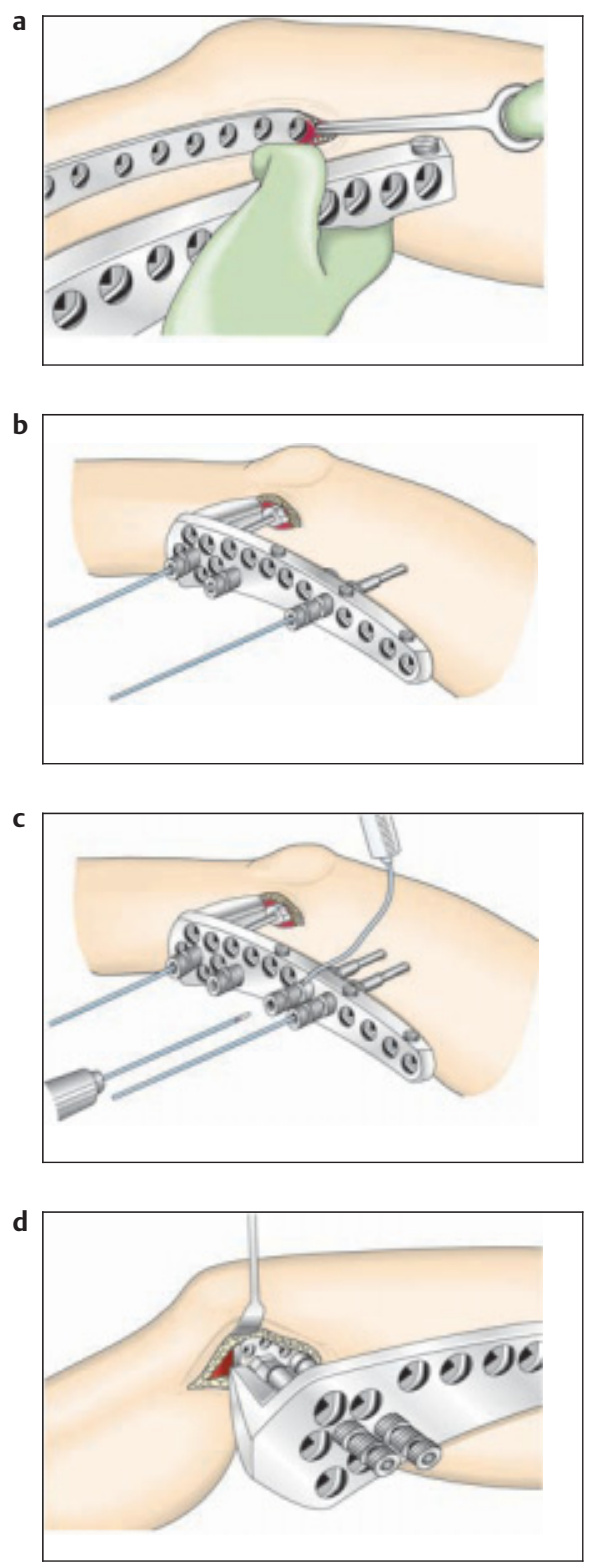

tellare Arthrotomie (Abb. 6) [13] und der Zugang wird nach proximal zwischen dem M. rectus und vastus lateralis im Faserverlauf erweitert und nach distal bis zur Tuberositas tibiae. Zunächst erfolgt die Rekonstruktion des Kondylenmassivs, hierzu wird die Patella nach medial gehalten. Die Femurkondylen können dann unter guter Sicht rekonstruiert werden [12].

Auch bei der LISS muss für die Platzierung der Zugschrauben des Kondylenmassivs die vorgesehene Lage des LISS antizipiert werden. Die Reposition des Femurschaftes mit dem Gelenkblock erfolgt durch eine indirekte Repositionstechnik, wobei auch ein Distraktor oder temporäre externe Stabilisierung zu Hilfe genommen werden kann (Abb.11).
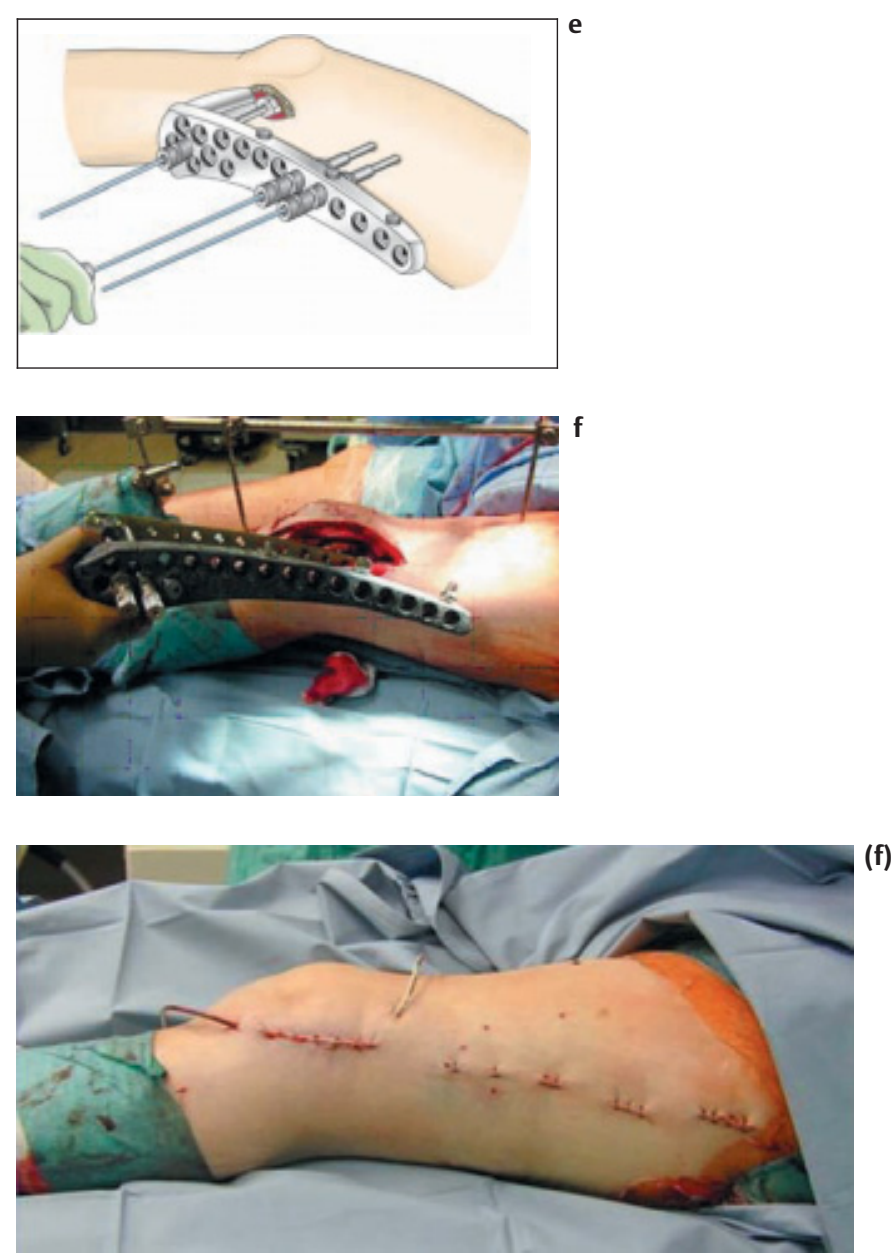

Abb.12 (a) Mithilfe eines Zielbügels wird das LISS perkutan unter Knochenkontakt zwischen Tractus iliotibialis und Periost eingebracht, (b) wobei die LISS distal 1,5 cm vor der Gelenklinie endet. Temporäre Fixierung der LISS am Kondylenblock und am Femurschaft mit einem K-Draht und (d) Einbringen der Schrauben (e) sowie Fixierung mit dem Drehmomentschlüssel. (f) Klinisches Beispiel.

Nach der Rekonstruktion des Gelenkblocks erfolgt in $30-50^{\circ}$ Knieflexion die Reposition des Kondylenblocks am Femurschaft und temporäre Fixierung mit 3,0 mm Drähten in gekreuzter Technik. Mithilfe eines Zielbügels wird das LISS dann zwischen Tractus iliotibialis und Periost eingebracht, wobei die LISS distal $1,5 \mathrm{~cm}$ vor der Gelenklinie endet. Beim perkutanen Vorschieben ist auf einen direkten Knochenkontakt zu achten (Abb.12). Nach Überprüfung der korrekten Lage mit dem Bildwandler in 2 Ebenen (Abb.13), erfolgt eine temporäre Fixierung der proximalen LISS zunächst am Kondylenblock und anschließend am Femurschaft mit einem K-Draht. Einbringen jeweils einer Schraube in das proximale sowie distale Fragment und Überprüfung der Reposition mit dem
Bildwandler in frontaler und sagittaler Ebene sowie auf Länge und Rotation. Die Reposition in der Frontalebene kann mit der Kabelmethode kontrolliert werden [13].

Dabei wird in Kniestreckung eine Verbindung zwischen Zentrum des Femurkopfes und die Talusmitte mit einem Elektrokauterkabel unter Bildwandlerkontrolle als Repräsentation der mechanischen Achse gebildet. Die koronare Ebene wird mittels des Winkels zwischen dorsaler Femurkortikalis und der Blumensaat'schen Linie und die Rotation über den Vergleich der Größe des rechten und linken Trochanter minor unter Bildwandler kontrolliert [14]. 

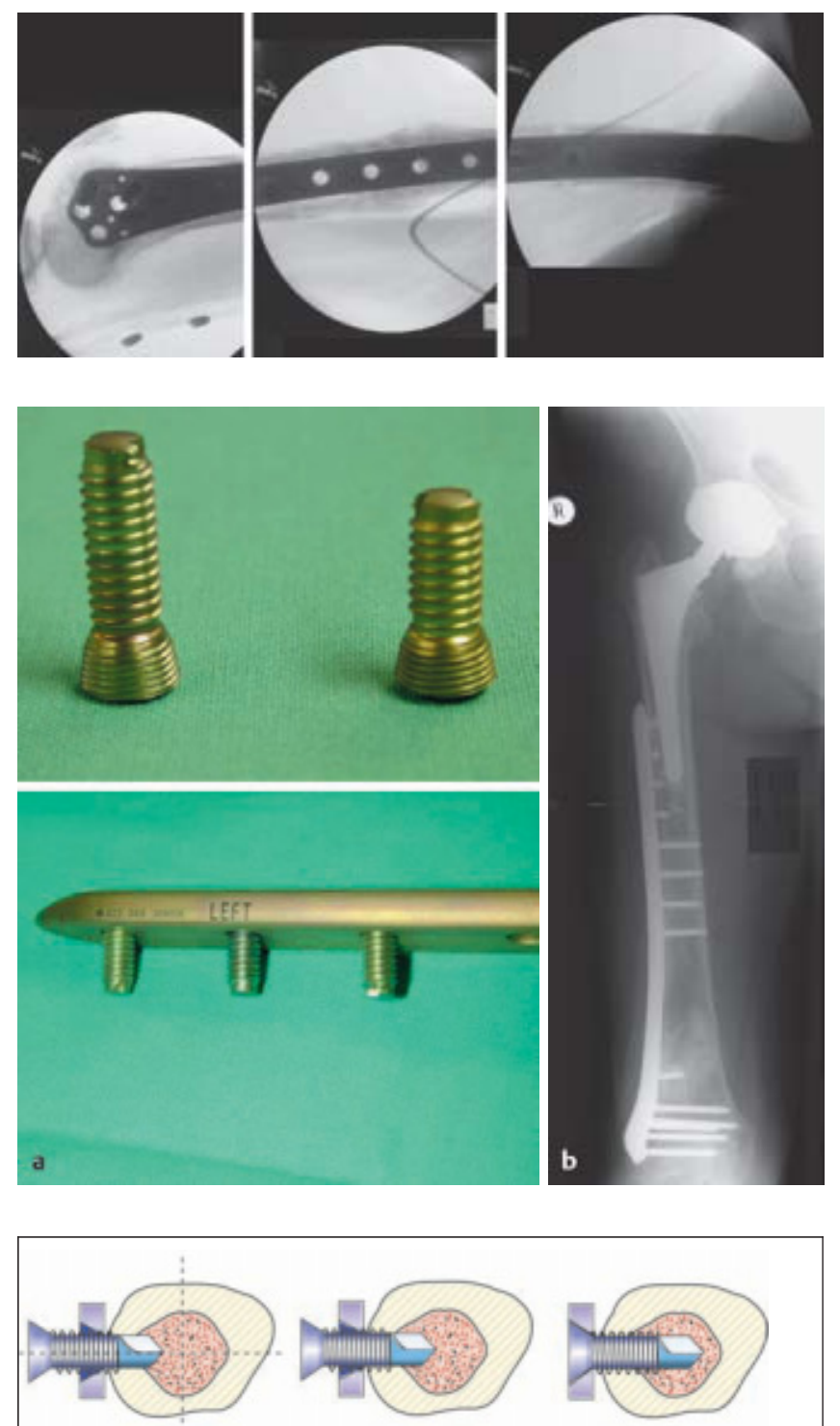

bohren

\section{Gewinde} schneiden
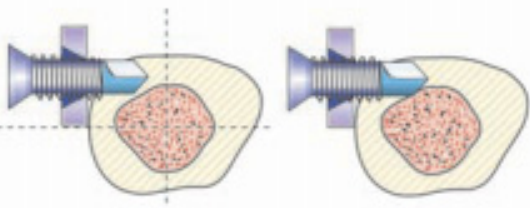

Gewinde schneiden
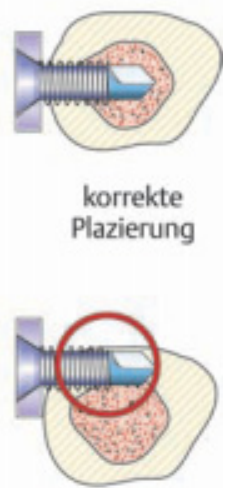

reduzierter Schraubenhalt
Abb. 14 (a) Bei periprothetischen Frakturen werden Schrauben ohne Bohrspitze (b) in Höhe der Endoprothese eingesetzt.

Abb.15 Durch die vorgegebene Bohrrichtung kommt es bei sagittaler Fehllage der LISS zu einer tangentialen Positionierung der monokortikalen Schraube im Femurschaft.

Durch diese tangentiale Schraubenlage können die Schrauben nur in einem kleinen Anteil nahe der Schraubenspitze verankert werden.
Durch ein zusätzliches Zuginstrumentarium kann eine Feinjustierung der Reposition erfolgen. Anschließend werden im proximalen sowie distalen Hauptfragment mindestens 4 Schrauben platziert.

Die langen distalen Schrauben im Bereich der Kondylen sollten parallel zum Tibiaplateau eingebracht werden, um eine korrekte Reposition des Kondylenfragmentes an den Femurschaft zu erzielen.

Auch bei liegenden Implantaten oder Endoprothesen kann die LISS erfolgreich eingesetzt werden [4], wobei in Höhe der Endoprothese Schrauben ohne Bohrspitze eingebracht werden (Abb.14).

Eine typische Komplikation ist der proximale Schraubenausriss, wobei eine nicht optimale Positionierung der LISS am Femurschaft und die monokortikale Fixation bei Osteoporose einen prädisponierenden Faktor darstellt. Durch die vorgegebene Bohrrichtung kommt es bei sagittaler Fehllage der LISS zu einer tangentialen Positionierung der monokortikalen Schraube im Femurschaft. Durch diese tangentiale Schraubenlage kann die Schrauben nur in einem kleinen Anteil nahe der Schraubenspitze verankert werden (Abb.15). In der Regel kommt es zu einem proximalen Schraubenausriss bei zunehmender Belastung der Extremität nach 6-8 Wochen (Abb.16). Neuere Biomechanische Untersuchungen ergeben, dass die mechanischen Belastungen bei Besetzten sämtlicher Schraubenoptionen einen hohen Stress auf die Frakturzone ausüben und somit die Stabilität der Gesamtkonstruktion zu rigide werden kann. Plattenbrüche sind dann die Folge (Abb.17). Aus diesem Grunde sollte, wenn möglich, in der Frakturzone über einen Bereich von 4-6 Löchern nicht fixiert werden.

\section{C) Platten-/Schraubenosteosynthese}

Bei partiell artikulären Frakturen (AO 33 B1 bis B3) kann eine isolierte Schraubenosteosynthese durchgeführt werden. Für sagittale Abscherfrakturen am lateralen (B1) oder medialen Kondylus (B2) eignen sich $6,5 \mathrm{~mm}$ Spongiosaschrauben mit einem $32 \mathrm{~mm}$ langen Gewinde. Alternativ kommen kanülierte Schrauben der Stärke 7,0 oder $7,3 \mathrm{~mm}$ zur Anwendung. Bei osteoporotischer Knochensubstanz empfiehlt sich die Verwendung von Unterlegscheiben, um eine Penetration der Kortikalis zu vermeiden. 

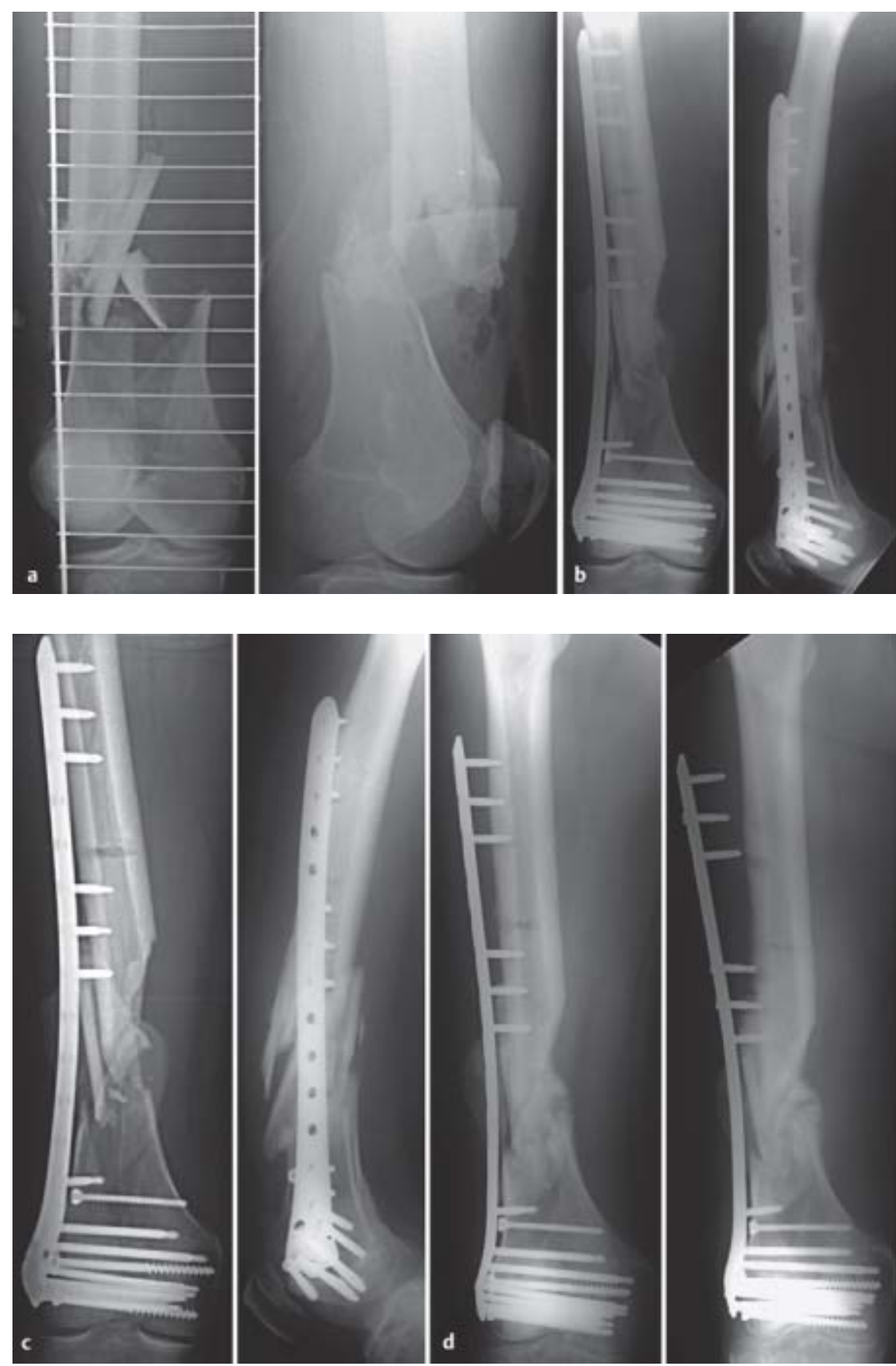

Abb. 16 Patient, 42 Jahre, distale Femurfraktur AO 33 C2, Osteosynthese 13-Loch-LISS mit proximaler Fehlage und sekundärem LISS-Ausriss.

Eine geschlossene Frakturreposition und perkutane (kanülierte) Schraubenosteosynthese ist anzustreben. Ein elegantes Verfahren ist die arthroskopisch assistierte geschlossene Frakturreposition. So können Knorpelverletzungen der Femurrolle genau bilanziert und Begleitverletzungen des Kniegelenkes (Meniskusund Bandläsionen) direkt diagnostiziert und ggf. therapiert werden.

Andere Autoren favorisieren ein limitiert offenes Vorgehen im Sinne eines medialen
Die Reposition und operative Versorgung einer Hoffa-Fraktur (B3) erfolgt in der Regel limitiert offen. Zur Osteosynthese bietet sich eine interfragmentäre Zugschraubenosteosynthese in ventrodorsaler Verlaufsrichtung an [16]. Auf eine subchrondrale Lage der Schraubenköpfe bzw. Positionierung der Schraubenköpfe außerhalb der Gelenkfläche ist zu achten. Hierbei kommen $3,5 \mathrm{~mm}$ oder $4,0 \mathrm{~mm}$ Spongiosa- bzw. Kortikalisschrauben zur Anwendung.

\section{D) Fixateur externe}

Bei höhergradigem Weichteilschaden oder polytraumatisierten Patienten kann eine temporäre Frakturstabilisierung mit einem Fixateur externe initial notwendig sein. Ein wesentlicher Vorteil des Fixateur externe ist die kurze OP-Zeit. Nachteilig ist zum einen der erforderliche Verfahrenswechsel, andererseits die potenzielle Gefahr einer Pintract-Infektion mit Ausbreitung in den Markraum.

Operationstechnisch werden die Pins über Stichinzisionen von anterolateral in das Femur eingebracht. Ist eine Transfixation des Kniegelenkes erforderlich, werden zusätzlich zwei Pins in der ventralen Tibia positioniert. Auf eine stabile Konstruktion ist zu achten, um die insbesondere bei der Lagerung auftretenden großen Hebelkräfte am Oberschenkel mit der Gefahr einer sekundären Frakturdislokation zu neutralisieren.

Eine anterolaterale Schanz-SchraubenPositionierung begünstigt das spätere Einschieben des Plattenfixateur-Systems, ohne Entfernung des externen Fixateurs.

Die Schanz-Schrauben sollten extraartikulär platziert werden, um der Gefahr eines Pintract-bedingten Gelenkempyems vorzubeugen.

\section{Nachbehandlung LISS und Nagel}

Das postoperative NachbehandlungsSchema erfolgt individuell nach Maßgabe des Operateurs. Prinzipielle Empfehlungen sind eine kurzzeitige Immobilisierung des Patienten und weichteilkonditionierende Maßnahmen sowie die Anwendung der Bewegungsschiene (CPM).

Die Mobilisierung der Patienten erfolgt in der Regel unter krankengymnastischer Anleitung mit einer Abroll- oder Teilbelastung der verletzten unteren Extremität mit $15-20 \mathrm{~kg}$ an zwei Unterarmgehstützen. In Abhängigkeit von den radiomorphologischen Durchbauungszeichen erfolgt eine sukzessive Belstungssteigerung bis zum Erreichen der Vollbelastung.

Bei Vorliegen von Begleitverletzungen wird das Nachbehandlungschema dementsprechend angepasst. 

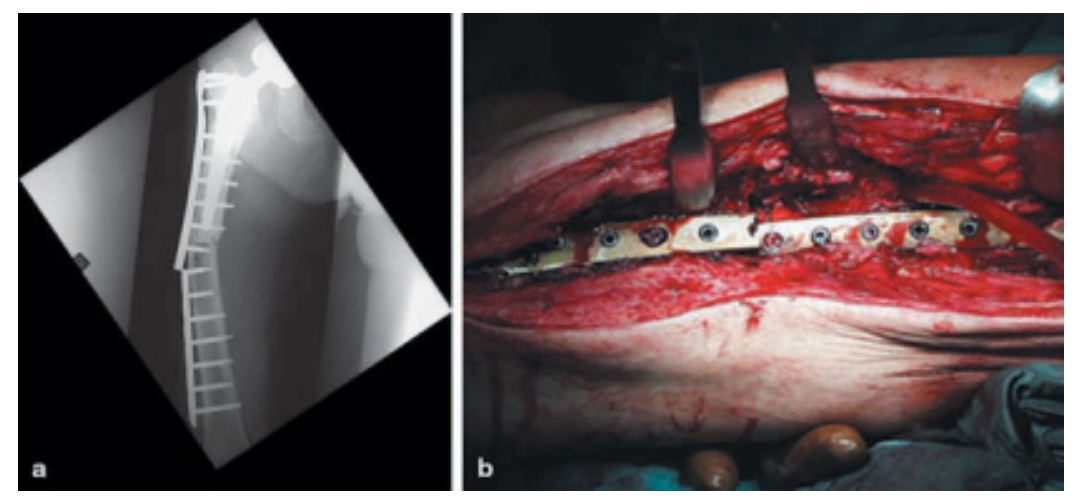

Abb.17 Bei Besetzung aller Schraubenlöcher erfolgt eine Stresskonzentration mit hoher Gefahr des (a) Plattenbruches, (b) intraoperativer Situs.

\section{Diskussion}

Distale und intraartikuläre Femurfrakturen des alten Menschen erfordern differenzierte operative Behandlungskonzepte.
Winkelstabile anatomisch vor geformte Platten-Fixationssysteme (LISS) sowie retrograde Marknägel weisen eine deutlich geringe Anzahl an Knochentransplantationen und Frakturheilungsstörungen gegenüber den offenen Verfahren auf $[3,5,20]$. Durch Schraubenlockerung und Scheibenwischereffekt zwischen Schrauben und Platte ist der Stabilitätsverlust bei den nicht winkelstabilen konventionellen Implantaten zu erklären [7].

Jedes der Systeme weist ein eigenes Komplikationsspektrum auf. Die Ursache des proximalen Schraubenausrisses bei der LISS können operationstechnische Fehler sein - einerseits durch Plattenfehllage und "Schraubenfixation“ in den Weichteilen bedingt - durch monokortikale Verankerung oder andererseits durch die Wahl zu kurzer Implantate verursacht sein. Eine Auslockerung im distalen Anteil der LISS am Kondylenmassiv ist selbst beim osteoporotischen Knochen bisher nicht beschrieben worden.

Kommentar in der Frühbesprechung: „Die Schraube hat aber „bombig“ gezogen“
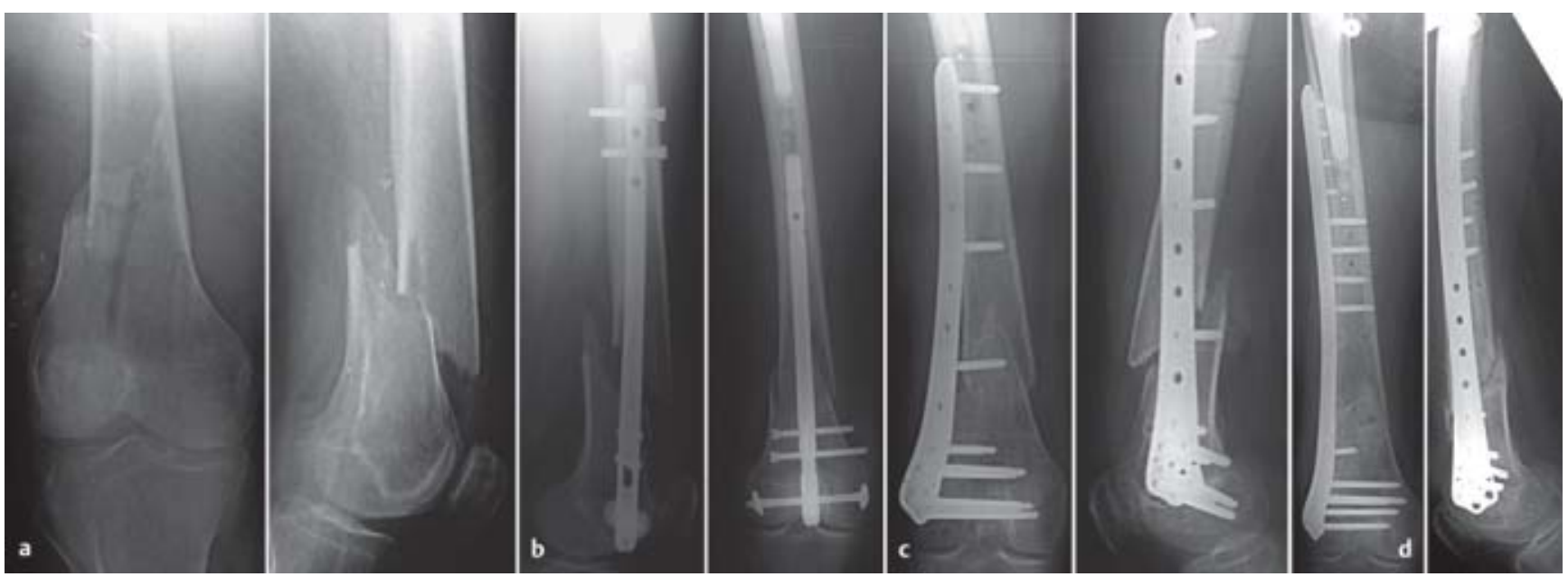

Abb.18 (a) 70-jährige Patientin mit distaler Femurfraktur AO 33 C1. (b) Retrograder Femurnagelung mit T2-Nagel mit mediolateraler Kondylenkompression, (c) Verfahrenswechsel auf 9-Loch-LISS bei Pseudarthrose, (d) erneuter Verfahrenswechsel auf 13-Loch-LISS bei verbliebener Valgusfehlstellung.
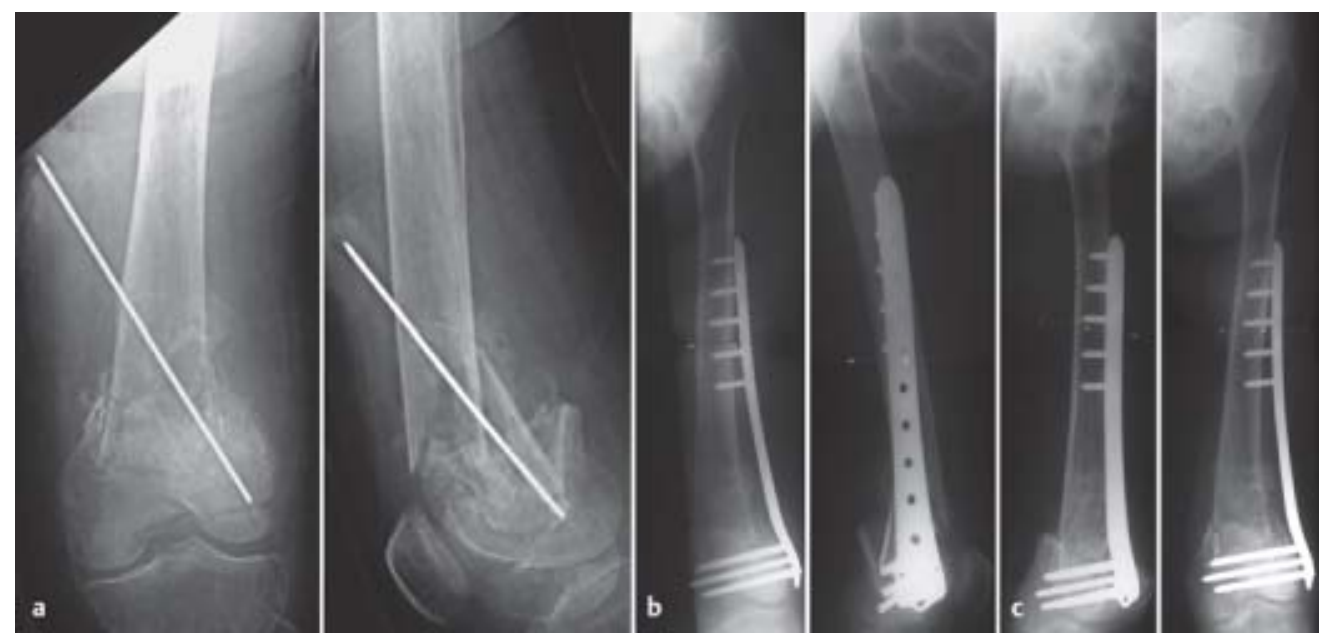

Abb. 19 88-jährige Patientin mit distaler Femurfraktur AO 33 A3, (a) Verlegung 3 Monate postoperativ bei Wundinfekt nach K-Drahtosteosynthese und Gipsimmobilisation. (b) Verfahrenswechsel auf 9-Loch-LISS nach 3 Monaten mit verbliebener Valgusfehlstellung,

(c) 3 Monate postoperativ. 

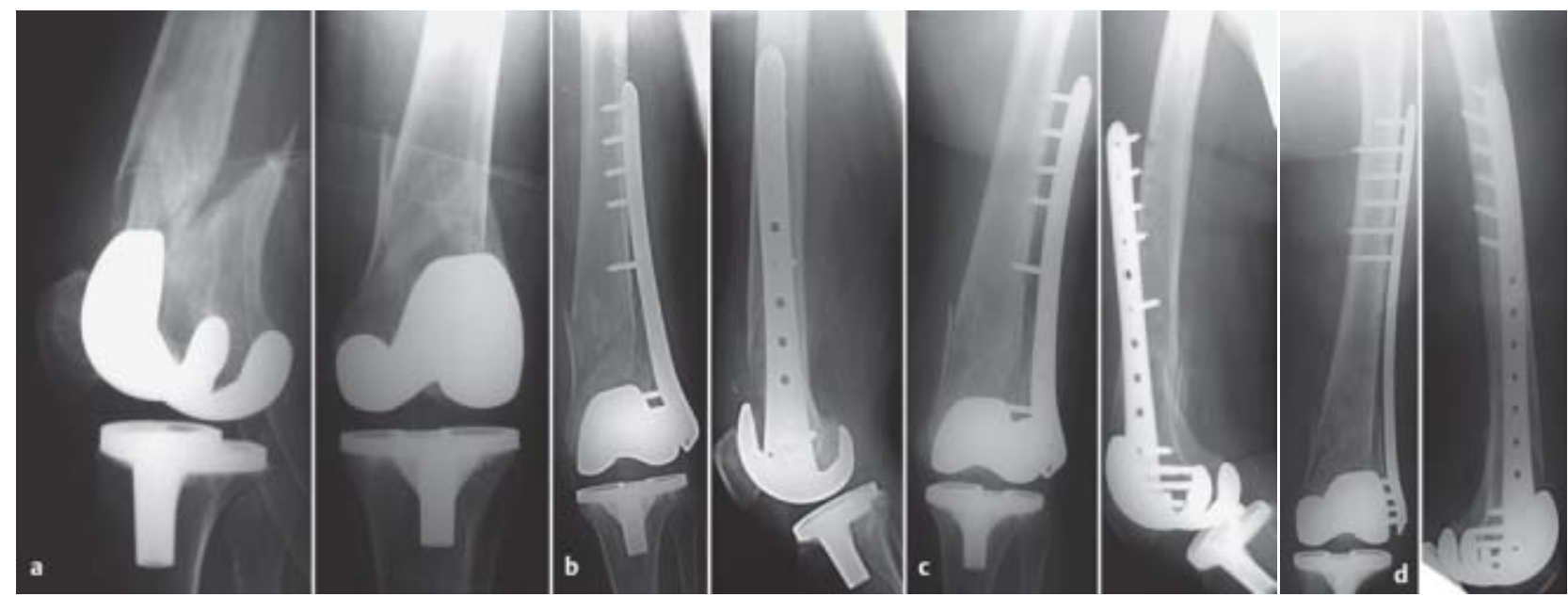

Abb.20 (a) Patientin, 80 Jahre, distale periprothetische Femurfraktur links bei Knie-TEP, (b) Osteosynthese mit 9-Loch-LISS und (c) sekundärem Ausriss, (d) Wechsel auf 13-Loch-LISS, 6 Monate postoperativ.
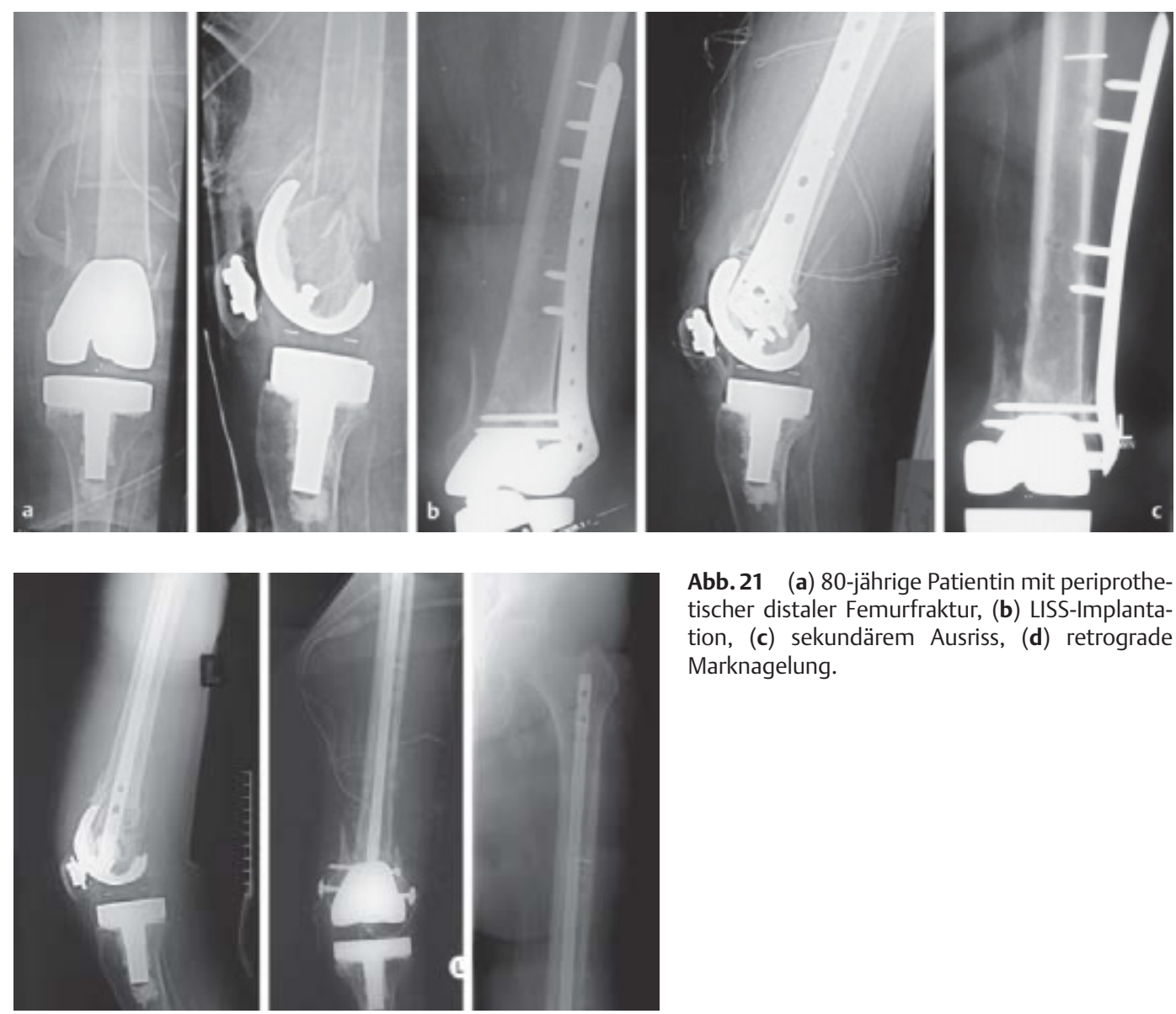

Abb. 21 (a) 80-jährige Patientin mit periprothetischer distaler Femurfraktur, (b) LISS-Implantation, (c) sekundärem Ausriss, (d) retrograde Marknagelung.
Aber auch intramedulläre Nägel haben unter wiederholter Belastung ähnliche Stabilitätsverluste gezeigt, wie die konventionellen Implantate [8]. Beim retrograden Marknagel ist das Implantatversagen durch eine distale Schraubenlockerung oder Auswanderung der distalen Verriegelungsbolzen geprägt. Die Spiralklinge ver- sucht dem entgegen zu wirken; zukünftige Weiterentwicklungen mit winkelstabilen Nagelsystemen können hier zu weiteren Verbesserungen beitragen.

Die Ausgangsbedingungen - besonders bei alten Menschen - stellen ein besonderes Dilemma dar:
Einerseits liegt meist eine veränderte, osteoporosebedingt geschwächte Knochensubstanz vor, die eine stabile Verankerung der Implantate erschweren, andererseits kann der alte Mensch im Rahmen der Nachbehandlung die verletzte Extremität nicht zuverlässig entlasten. Weiter erschwerend kommt hinzu, dass die neu- 
en Stabilisierungssysteme bei minimalinvasiver Implantationstechnik auf eine gute intraoperative Röntgendarstellung angewiesen ist, die bei höhergradiger Osteoporose häufig nicht zuverlässig gelingt.

Umso wichtiger ist die dezidierte präoperative Frakturanalyse und OP-Planung, ggf. auch die Bereitstellung oder aufwändige Organisation geeigneter Alternativimplantate oder sogar die Weiterverlegung in eine andere Klinik.

Ob dem retrograden Femurnagel oder winkelstabilen Plattensystemen der Vorzug gegeben werden sollte, hängt wesentlich von der individuellen Frakturanatomie und der Erfahrung des Operateurs ab. Hier dargestellte Fälle zeigen Beispiele, bei denen das eine Verfahren versagte und mit der Alternativmethode zur Ausheilung gebracht werden konnte.

Systemunabhängig ist die operative Versorgung eine distalen oder intraartikulären Femurfraktur bei osteoporotischer Knochenstruktur eine technisch sehr anspruchvolle Operation. Eine generelle Empfehlung für ein Implantat oder eine bestimmte operative Vorgehensweise kann nicht ausgesprochen werden. Ziel muss in jedem Fall sein, den Patienten übungs- oder sogar belastungsstabil zu versorgen.

\section{Literatur}

${ }^{1}$ El Maraghy AW, Schemitsch EH, Richards RR. Femoral and cruciate blood flow after retrograde femoral reaming: a canine study using laser Doppler flowmetry. J Orthop Trauma 1998; 12: $253-258$

${ }^{2}$ Grass R, Biewener A, Rammelt S, Barthel S, Zwipp H. Klinische Erprobung des distalen Femurnagels. Unfallchirurg 2002; 105: $783-790$

${ }^{3}$ Grass R, Zwipp H. Minimally invasive method for treatment of supra-diacondylar femoral fractures. Zentralbl Chir 1998; 123: $1247-1251$
${ }^{4}$ Hockertz TJ, Gruner A, Reilmann H. Treatment of femoral fracture after total knee arthroplasty with the LIS system: a new method. Unfallchirurg 1999; 102: $811-814$

${ }^{5}$ Janzing HM, Stockman B, Van Damme G, Rommens P, Broos PL. The retrograde intramedullary supracondylar nail: an alternative in the treatment of distal femoral fractures in the elderly? Arch Orthop Trauma Surg 1998; 118: $92-95$

${ }^{6}$ Janzing HM, Vaes F, Van DG, Stockman B, Broos PL. Treatment of distal femoral fractures in the elderly. Results with the retrograde intramedullary supracondylar nail. Unfallchirurgie 1998; 24: 55-59

${ }^{7}$ Koval KJ, Hoehl JJ, Kummer FJ, Simon JA. Distal femoral fixation: a biomechanical comparison of the standard condylar buttress plate,a locked buttress plate, and the 95-degree blade plate. J Orthop Trauma 1997; 11: $521-524$

${ }^{8}$ Koval KJ, Kummer FJ, Bharam S, Chen D, Halder S. Distal femoral fixation: a laboratory comparison of the 95 degrees plate, antegrade and retrograde inserted reamed intramedullary nails. J Orthop Trauma 1996; 10: $378-382$

${ }^{9}$ Krettek C, Miclau T, Grün O, Schandelmaier $\mathrm{P}$, Tscherne $\mathrm{H}$. Intraoperative control of axes, rotation and length in femoral and tibial fractures-technical note. Injury 1998; 29 [Suppl]: 29-39

10 Krettek C, Schandelmaier P, Lobenhoffer HP, Tscherne H. Komplextrauma des Kniegelenkes - Diagnostik, Management und Therapieprinzipien. Unfallchirurg 1996; 99: 616-628

${ }^{11}$ Krettek C, Schandelmaier P, Miclau T, Bertram $H$, Tscherne $H$. Transarticular joint reconstruction and indirect plate osteosynthesis for severe distal intercondylar C2/C3 type femoral fractures. Injury 1997; 28 [Suppl]: $31-41$

${ }^{12}$ Krettek C, Schandelmaier P, Miclau T, Tscherne H. Minimally invasive percutaneous plate osteosynthesis (MIPPO) using the DCS in proximal and distal femoral fractures. Injury 1997; 28 [Suppl]: 20-30

${ }^{13}$ Krettek C, Schandelmaier P, Tscherne H. Distale Femurfrakturen: Transartikuläre Rekonstruktion, perkutane Plattenosteosynthese und retrograde Nagelung. Unfallchirurg 1996; 99: 2-10

${ }^{14}$ Krettek C, Schulte Eistrup S, Schandelmaier $\mathrm{P}$, Rudolf J, Tscherne H. Osteosynthese von Femurschaftfrakturen mit dem unaufgebohrten AO- Femurnagel (UFN). Operative Technik und erste klinische Ergebnisse mit Standardverriegelung. Unfallchirurg 1994 97: 549-567
${ }^{15}$ Lucas SE, Seligson D, Henry SL. Intramedullary supracondylar nailing of femoral fractures. A preliminary report of the GSH supracondylar nail. Clin Orthop 1993; $200-$ 206

${ }^{16}$ Meyer C, Enns P, Alt V, Pavlidis T, Kilian O, Schnettler R. Die Problematik der HoffaFraktur. Unfallchirurg 2004; 107: 15-21

${ }^{17}$ Mize RD, Bucholz RW, Grogan DP. Surgical treatment of displaced, comminuted fractures of the distal end of the femur. J Bone Joint Surg Am 1982; 64: 871 -878

18 Müller ME, Nazarian S, Koch P, Schatzker J. The comprehensive classification of fractures of long bones. Springer Verlag Heidelberg (1990)

${ }^{19}$ Neer CS, Grantham SA, Shelton ML. Supracondylar fracture of the adult femur. A study of one hundred and ten cases. J Bone Joint Surg Am 1967; 49: 591 -613

${ }^{20}$ Ostermann PA, Hahn MP, Ekkernkamp A, David A, Muhr G. Retrograde interlocking nailing of distal femoral fractures with the intramedullary supracondylar nail. Chirurg 1996; 67: 1135-1140

${ }^{21}$ Schandelmaier P, Stephan C, Krettek C, Tscherne H. Distale Femurfrakturen. Unfallchirurg 2000; 70: 428-436

${ }^{22}$ Schandelmaier P, Krettek C, Miclau T, Stephan C, Könemann B, Tscherne H. Stabilization of distal femoral fractures using the LISS. Techn Orthop 1999; 14: 230-246

${ }^{23}$ Tscherne H, Oestern HJ, Trentz O. Spätergebnisse der distalen Femurfraktur und ihre besonderen Probleme. Zentralbl Chir 1977; $102897-904$

Dr. med. Uwe Frerichmann

Oberarzt

Dr. med. Alexander Rübberdt

Oberarzt

Univ.-Prof. Dr. med. Michael J. Raschke

Direktor der Klinik

Klinik für Unfall-, Hand- und Wiederherstellungschirurgie

Universitätsklinikum Münster

Waldeyerstr. 1

D-48149 Münster 